\title{
Multi-excitonic complexes in single InGaN quantum dots
}

\author{
R. Seguin*, S. Rodt, A. Strittmatter, L. Reißmann, T. Bartel, A. Hoffmann, and D. Bimberg \\ Institut für Festkörperphysik, Technische Universität Berlin, D-10623 Berlin, Germany \\ E. Hahn and D. Gerthsen \\ Laboratorium für Elektronenmikroskopie, Universität Karlsruhe, D-76128 Karlsruhe, Germany
}

\begin{abstract}
Cathodoluminescence spectra employing a shadow mask technique of InGaN layers grown by metal organic chemical vapor deposition on $\mathrm{Si}(111)$ substrates are reported. Sharp lines originating from InGaN quantum dots are observed. Temperature dependent measurements reveal thermally induced carrier redistribution between the quantum dots. Spectral diffusion is observed and was used as a tool to correlate up to three lines that originate from the same quantum dot. Variation of excitation density leads to identification of exciton and biexciton. Binding and anti-binding complexes are discovered.
\end{abstract}

The fundamental processes responsible for optical recombination in InGaN/GaN quantum structures are a matter of controversial discussion. Wurzite GaN-based semiconductors exhibit strong piezoelectric fields owing to their large piezoelectric constants. In thin layers these fields give rise to a blueshift of the transition energy with increased excitation density due to screening of the quantum confined Stark effect. ${ }^{1}$ Yet, the quantum dot (QD) nature of compositional fluctuations in the InGaN layers can lead to similar effects. ${ }^{2,3}$ The purpose of the present work is to demonstrate three-dimensional confinement of carriers in InGaN by conducting spatially high-resolved cathodoluminescence (CL) measurements. We observe a multitude of sharp lines, several of these lines unambiguously originating from the same QD. Temperature and excitation density dependent CL investigations of these lines give further proof for the existence of strong localization and lead to the distinction of excitonic and biexcitonic recombination.

The samples were grown by low-pressure metal organic chemical vapor deposition using a horizontal AIX200 RF reactor. Prior to loading the reactor the substrates were treated by wet chemical etching yielding an oxide-free, and hydrogen terminated $\mathrm{Si}(111)$ surface. An AlN layer acting as nucleation surface was obtained by using a previously described conversion process of AlAs to AlN. ${ }^{4}$ In the following step $\mathrm{Al}_{0.05} \mathrm{Ga}_{0.95} \mathrm{~N} / \mathrm{GaN}$ buffer layers were grown at $\mathrm{T}=1150{ }^{\circ} \mathrm{C}$ up to a total thickness of $1 \mu \mathrm{m}$. The InGaN layers were grown at $800{ }^{\circ} \mathrm{C}$ using TMGa, TMIn, and ammonia as precursors. Total pressure was kept at 400 mbar during InGaN deposition. The growth was finished with a $20 \mathrm{~nm}$ GaN cap layer grown during heatup to $1100{ }^{\circ} \mathrm{C}$.

The samples were investigated with a JEOL JSM 840 scanning electron microscope equipped with a cathodoluminescence setup. ${ }^{5}$ All measurements were made at a temperature of $6.5 \mathrm{~K}$ unless stated otherwise. The low temperatures were obtained by mounting the samples

*email: seguin@sol.physik.tu-berlin.de onto a He flow cryostat. The luminescence light was dispersed by a $0.3 \mathrm{~m}$ monochromator equipped with a 2400 lines/mm grating and detected with a nitrogen cooled SiCCD camera, providing a spectral resolution of $310 \mu \mathrm{eV}$ at $3 \mathrm{eV}$. In order to increase spatial resolution we applied metal shadow masks onto the sample surface with aperture diameters of 100 and $200 \mathrm{~nm}$.

The integral CL spectrum shows an intensive peak centered at $2.98 \mathrm{eV}$ with a full width at half maximum (FWHM) of $80 \mathrm{meV}$ which originates from the InGaN layer. Weak luminescence of the donor bound GaN exciton can be observed at $3.46 \mathrm{eV}$.

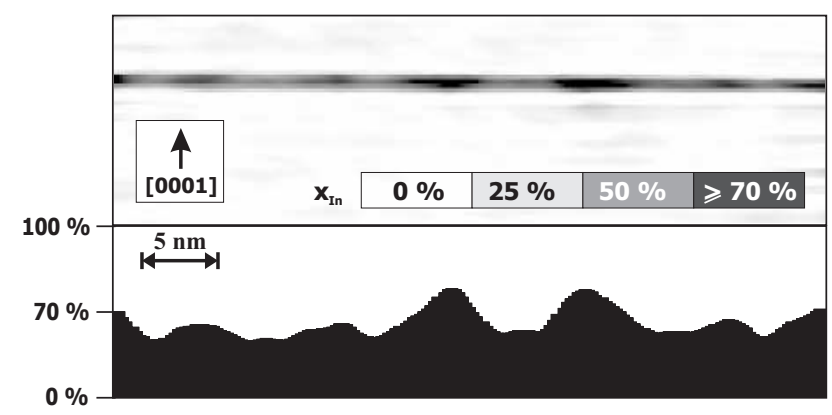

FIG. 1: DALI processed cross section HRTEM image. The upper image shows a gray-scale coded map of the local In concentrations in the sample. The InGaN layer is about $2 \mathrm{~nm}$ in height and shows prominent In fluctuations. The lower image visualizes the In concentration along the layer.

When measured through one of the apertures the InGaN peak decomposes into sharp lines. Recently, similar observations on InGaN structures grown on sapphire have been reported by other groups using $\mu$-PL. ${ }^{6,7}$ The narrowest lines show a FWHM of $0.48 \mathrm{meV}$. These lines can be found over a wide range of energies $(2.8-3.2 \mathrm{eV})$ thus covering the whole ensemble peak. Since the line density is very high we mainly investigated the high and low energy sides of the ensemble peak where single lines are well resolved.

$\mathrm{DALI}^{8}$ (digital analysis of lattice images) processed cross-section high-resolution TEM (HRTEM) measurements, performed under short times of irradiation $(\ll 1$ 
min) to prevent electron beam induced artefacts, ${ }^{9}$ show alternating areas of high and low In content. The In-rich domains have a lateral size of about $5 \mathrm{~nm}$ (Fig. 11). Such fluctuations are small enough to provide strong localization of carriers and are identified as the source of the sharp peaks. A similar growth mode of QDs has been reported for II-VI compounds, such as CdSe/ZnSe. There, composition fluctuations induce strong carrier localization and QD-like behavior. ${ }^{10}$

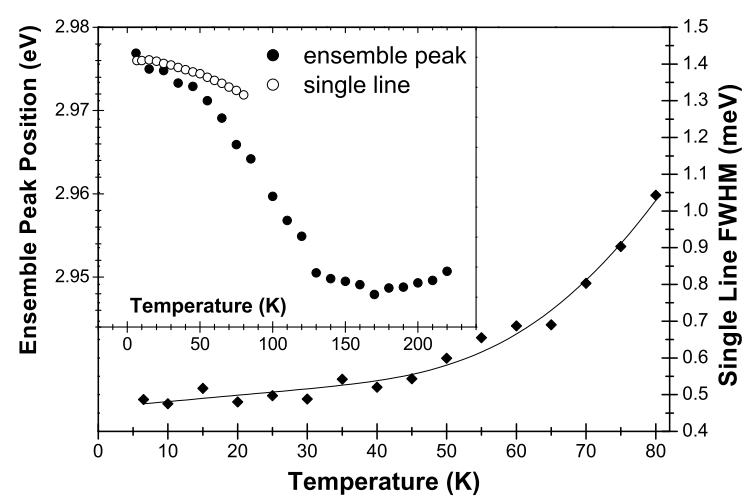

FIG. 2: Temperature dependent measurements: FWHM of a single line with a fit according to Eq. (1). The inset shows the energetic positions of the ensemble peak and of a single line. The single line position has an offset for easier comparison.

Temperature dependent measurements of single lines were conducted. The lines were visible in a temperature range from 6 to $80 \mathrm{~K}$. At higher temperatures the lines broaden and the peak intensity is too low to resolve them any more. The energetic position of single lines shifts about $4 \mathrm{meV}$ to the red with increasing temperature (inset Fig. 2]). In the present case (Fig. 21) the FWHM $\Gamma(T)$ increased from initially $0.48 \mathrm{meV}$, which is slightly broader than our resolution limit, to about $1.05 \mathrm{meV}$, at a rate well below $k T . \Gamma(T)$ was fitted well using

$$
\Gamma(T)=\Gamma_{0}+\gamma_{p} T+\gamma_{a} \exp \left(-\frac{E_{A}}{k_{B} T}\right) .
$$

$\Gamma_{0}$ describes the initial FWHM due to the limited spectral resolution and the spectral diffusion as will be discussed later. The linear term describes acoustic phonon interaction. The third term describes dephasing due to excitation of the carriers into the surrounding InGaN layer. Optical phonons are negligible here due to their energy of $91.5 \mathrm{meV}$ in $\mathrm{GaN}$, which is much larger than $k T$ at $6-80 \mathrm{~K}$. The fit yields coupling constants of $\gamma_{p}=1.7 \pm 0.7 \mu \mathrm{eVK}^{-1}, \gamma_{a}=36 \pm 25 \mathrm{meV}$ and an activation energy of $E_{A}=31 \pm 5 \mathrm{meV}$. This gives us an estimate of the localization depth of the probed QD relative to the surrounding InGaN layer.

Temperature dependent PL measurements of the ensemble peak were made as well. As can be seen in the inset of Fig. 2] the red shift of the ensemble peak is initially larger than that of a single line. This behavior is attributed to preferential quenching of luminescence from small QDs emitting light at the high energy side of the spectrum. As there are also weakly localizing QDs in the InGaN layer, the quenching sets in at lower temperatures compared to, e.g., InAs/GaAs QDs. ${ }^{11}$ Since this is an ensemble effect it cannot be observed for the single line. At higher temperatures thermal activation of carriers into higher energetic states completely compensates the redshift of the bandgap. This results in an s-shape of the data points as is characteristic for $\mathrm{QD}$ ensembles.

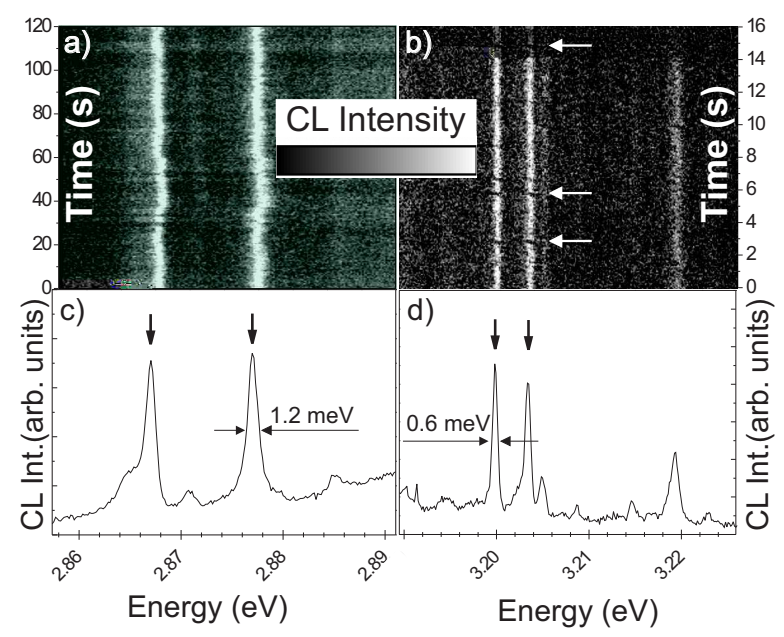

FIG. 3: Time series: (a) 400 spectra with $300 \mathrm{~ms}$ integration time each. Spectral diffusion is visible and correlates the marked lines in (c). The lines are broadened significantly due to the jitter. (b) 200 spectra with $80 \mathrm{~ms}$ integration time each. On/off blinking (indicated by the white arrows) is visible and correlates the marked lines in (d). (c) and (d) show averaged spectra of the time series above.

We took spectra of single lines with short integration time to generate time series. One series consists of several 100 spectra with fixed integration times between 80 and $300 \mathrm{~ms}$ each. We observe a slight stochastic variation of the peak energies [Fig. [3(a)], known as spectral diffusion. ${ }^{12}$ The QDs experience fluctuating electric fields which cause a slight shift of the energy level in the QD via the quantum confined Stark effect. The source of these fields is charging and decharging of nearby defects or interface states. When longer integration times are used, spectral diffusion contributes significantly to the observed linewidth [Fig. 31(c)] giving rise to a seemingly larger linewidth than our resolution limit even at low temperatures. At short integration times, on/off blinking of the lines can be observed as well [Fig. [3(b)]. The internal electric fields spatially separate electrons and holes and reduce the wavefunction overlap or cause the carriers to leave the QD completely. Strong fields from nearby centers can thus quench the luminescence completely. ${ }^{13}$

Both effects can be used to correlate transitions that originate from the same QD since they experience the 
same electric fields and hence the lines exhibit the same energetic jitter and blinking behavior. ${ }^{14}$ We observed doublets and on rare occasions triplets that show a similar jitter. Typical energy differences vary between 2 and $20 \mathrm{meV}$. The presence of such groups demonstrates the existence of higher excitonic complexes in one QD.

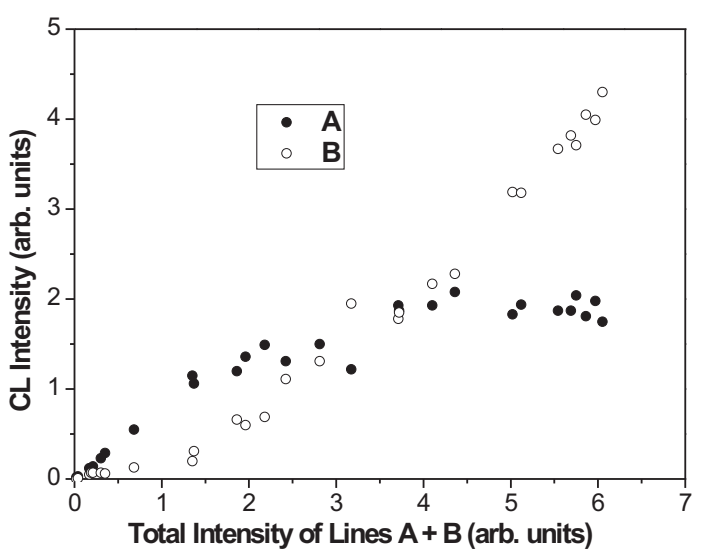

FIG. 4: Excitation density dependence. The total intensity equals the local excitation density. Line A dominates at low excitation densities and then saturates. Line B takes over at high excitation densities.

In Fig. 4 the intensity of two lines A and B which had been correlated by their jitter pattern is plotted versus the total intensity of both lines. The total intensity is a good measure for the local excitation density, assuming that the QD does not saturate and no other lines with similar jitter are present. Line A depends linearly on the excitation density at low densities and then saturates. Line B depends quadratically on the excitation density and dominates in the high excitation regime. This behavior is typical for exciton and biexciton. ${ }^{15}$ At low excitation densities the probability of two electron-hole pairs populating the same QD to form a biexciton before one of them recombines is low. At high excitation densities the probability increases until it is more likely to find biexcitons in the QD than excitons. Biexcitons have not yet been reported for InGaN QD structures before. In the particular case of Fig. 4 the biexciton binding energy, which is generally measured relative to the exciton recombination energy, is $-3.5 \mathrm{meV}$.

B-type lines could be observed at higher and lower energies with respect to corresponding A-type lines depending on the particular QD examined. We found values ranging from -3.5 to $16 \mathrm{meV}$. Hence binding energies can be both positive and negative. "Anti-binding" complexes do not exist in bulk semiconductors. In QDs, they can be stable due to the localizing confinement potential. ${ }^{16}$ Further investigation of the multi-excitonic complexes are under way.
In conclusion, we have demonstrated QD-like behavior of the luminescence originating from a thin InGaN layer. Composition fluctuations within the layer identified via HRTEM are the source for sharp lines in the spectrum. Temperature dependent measurements further prove strong localization within the layer. Observation of spectral diffusion and blinking has been used to demonstrate the existence of multi-excitonic states. Excitation density dependent measurements unveiled lines with excitonic and biexcitonic origin. Binding and antibinding complexes could be observed.

Part of this work has been funded by Sonderforschungsbereich 296 of Deutsche Forschungsgemeinschaft.

${ }^{1}$ A. Hangleiter, J. Im, H. Kollmer, S. Heppel, J. Off, and F. Scholz, MRS Internet J. Nitride Semicond. Res. 3, 15 (1998).

2 S. Chichibu, T. Azuhata, T. Sota, and S. Nakamura, Appl. Phys. Lett. 69, 4188 (1996).

${ }^{3}$ I. L. Krestnikov, N. N. Ledentsov, A. Hoffmann, and D. Bimberg, Phys. Rev. B 66, 155310 (2002).

4 A. Strittmatter, A. Krost, M. Straßburg, V. Türck, D. Bimberg, J. Bläsing, and J. Christen, Appl. Phys. Lett. 74, 1242 (1999).

5 M. Grundmann, J. Christen, N. N. Ledentsov, J. Böhrer, D. Bimberg, S. S. Ruvimov, P. Werner, U. Richter, U. Gösele, J. Heydenreich, V. M. Ustinov, A. Yu. Egorov, A. E. Zhukov, P. S. Kop'ev, and Zh. I. Alferov, Phys. Rev. Lett. 74, 4043 (1995)

${ }^{6}$ O. Moriwaki, T. Someya, K. Tachibana, S. Ichida, and Y. Arakawa, Appl. Phys. Lett. 76, 2361 (2000).

7 R. A. Oliver, G. A. D. Briggs, M. J. Kappers, C. J. Humphreys, S. Yasin, J. H. Rice, J. D. Smith, and R. A. Taylor, Appl. Phys. Lett. 83, 755 (2003).

${ }^{8}$ D. Gerthsen, E. Hahn, B. Neubauer, V. Potin, A. Rosenauer, M. Schowalter, Phys. Stst. Sol. (c) 0, 1668 (2003)

9 T. M. Smeeton, M. J. Kappers, J. S. Barnard, M. E. Vickers, and C. J. Humphreys, Appl. Phys. Lett. 83(26), 5419 (2003).

10 I. L. Krestnikov, M. Straßburg, M. Caesar, A. Hoffmann, U. W. Pohl, and D. Bimberg, Phys. Rev. B 60, 8695 (1999).

${ }^{11}$ R. Heitz, I. Mukhametzhanov, A. Madhukar, A. Hoffmann, and D. Bimberg, J. Electron. Mater. 28(5), 520 (1999).

12 S. A. Empedocles, D. J. Norris, and M. G. Bawendi, Phys. Rev. B 77, 3873 (1996).

13 P. Castrillo, D. Hessman, M.-E. Pistol, and J. A. Prieto, Jpn. J. Appl. Phys. 36, 4188 (1997).

14 V. Türck, S. Rodt, O. Stier, R. Heitz, R. Engelhardt, U. W. Pohl, and D. Bimberg, Phys. Rev. B 61, 9944 (2000).

15 M. Grundmann and D. Bimberg, Phys. Rev. B 55, 9740 (1997).

16 S. Rodt, R. Heitz, A. Schliwa, R. L. Sellin, F. Guffarth, and D. Bimberg, Phys. Rev. B 68, 035331 (2003). 